\title{
Synthesis of Uncorrelated Acoustic Quality Evaluation Indices of Buildings
}

\author{
K. KOSAŁA*
}

AGH - University of Science and Technology, Faculty of Mechanical Engineering and Robotics

Department of Mechanics and Vibroacoustics, al. A. Mickiewicza 30, 30-059 Krakow, Poland

\begin{abstract}
Previous studies and analyses conducted up to now on the indicatory description of the acoustic quality of buildings concerned single value indices based on several selected acoustic parameters that are correlated with each other. Transformation of values, correlated into a new set of independent components for the purpose of their future synthesis, took place using the Singular Value Decomposition (SVD) technique. In this article, it is shown that SVD can also be applied to the determination of the single-value global acoustic quality index in the case where the decomposed index observation matrix contains partial indices that are not only correlated, but also uncorrelated. Alternatively, a statistical method used in econometrics, i.e. Comparative Multivariate Analysis (CMA), was adapted to obtain a single-value index from uncorrelated partial indices. Both proposed methods of synthesis have been verified using the example of a group of buildings - Roman Catholic churches.
\end{abstract}

DOI: $10.12693 /$ APhysPolA.123.1064

PACS: 43.55.-n, 43.55.+p

\section{Introduction}

The correct acoustic utilisation of the interior of a building is related to its function and purpose. Depending on function of the buildings, the scope of acoustic production may vary. Examples of public buildings with a narrow range of acoustic production are auditoriums and theatres or religious interiors such as synagogues, where speech signals are dominant. Another example is the group of buildings with acoustic conditions that are appropriate for playing and listening to music exclusively, which includes concert halls. A different specific group of buildings has multi-functional interiors with a wide range of acoustic production, including music and speech. These are buildings such as: opera houses, movie theatres and religious buildings, particularly Roman Catholic churches. Interiors of this type are a challenge for designers, because they require compromises of acoustic conditions.

The methods of Beranek [1] and Ando [2] are acknowledged methods of acoustic evaluation of concert halls and opera houses. The components of single-value ratings in these methods are uncorrelated with each other and describe various aspects of the acoustic field [3]. Acoustic evaluation of rooms may also take place using the more easily applicable impulse method. Acoustic parameters are calculated from the studied impulse response, which, in comparison with preferred values obtained from subjective studies, constitute the basis for acoustic evaluation. These parameters are correlated with one another; thus, it is difficult to use them to evaluate the acoustics of an interior in a complete way, e.g. by using one value, if even for the reason of certain information being duplicated during synthesis of these parameters.

\footnotetext{
*e-mail: krzysztof.kosala@agh.edu.pl
}

Another approach to evaluation of the acoustic quality of buildings has been proposed - the global evaluation, based on partial indices related to the acoustic functions and purpose of a given building [4]. The global acoustic quality index of a building, $W_{G}$, is a function of many partial indices,

$$
W_{G}=f\left(W_{1}, W_{2}, W_{3}, \ldots, W_{n}\right),
$$

where $W_{1}, \ldots, W_{n}$ are partial indices.

The global index is an approximated index and the general measure for evaluation. Partial evaluation indices give more accurate information on the acoustic properties of a building. All partial indices and the global index take values within the interval from 0 to 1 . A value of 0 signifies bad acoustic properties of the building, significantly deviating from preferred values, and the value of 1 signifies good acoustic properties, in accordance with the preferred values of acoustic parameters.

The singular value decomposition (SVD) technique was applied to solve the problem of correlated acoustic indices in a single-value evaluation [5]. Up to this point, application of SVD for construction of a single-value index $W_{l}$ from selected correlated partial indices took place by using the so-called full correlation [6] or decorrelation of the index matrix of observation [7].

This article shows the next step in studies on the index method of acoustic quality evaluation of buildings which is a synthesis of uncorrelated indices. Utilisation of two tools is proposed to fulfil this purpose - the SVD technique and the statistical method of comparative multivariate analysis (CMA). Here, the SVD technique will be applied for decomposition of the observation matrix of all partial indices used for evaluation that may be correlated or uncorrelated. The CMA method may be applied only in the case of uncorrelated indices. For this purpose, it is necessary to separate the partial indices taken for evaluation into strongly correlated and uncorrelated indices. The single value local index $W_{l}$ will be created from strongly correlated indices using the SVD method 
which, together with other uncorrelated indices, will be a component of the global index $W_{G}$.

Both proposed methods of synthesis have been verified using the example of a group of buildings - Roman Catholic churches. It was verified which one of the global indices, $W_{G s}$ or $W_{G d}$, was better correlated with the initial partial indices.

\section{Statistical method of synthesis of uncorrelated indices}

The statistical method, i.e. CMA, adapted to construction of the global index $W_{G s}$, is related to the analysis of complex phenomena. A complex phenomenon is understood as an abstract concept that illustrates the directly immeasurable qualitative state of real objects, described by a certain number greater than one of the so-called diagnostic variables. Multi-dimensional methods of statistical analysis used in econometrics [8] make it possible to present variables (building properties) in such a form so that they can be directly compared to one another. It is possible to construct a ranking of buildings in the light of multi-criteria evaluations. According to the assumptions of this method, clarifying (diagnostic) variables should be: characterised by high variability; strongly correlated with the clarified variable; and weakly correlated with each other. Acoustic parameters are the clarified variables, while partial indices are the clarifying variables.

Adaptation of the statistical method for the purpose of construction of a global index will be based on the use of a method for determining the weights of variables (uncorrelated partial indices) and a method of variable aggregation. It is proposed that property variability coefficients are used for calculation of measures of relative informational value which will be the weights $\omega_{j}$ of variables

$$
\omega_{j}=\frac{V\left(X_{j}\right)}{\sum_{j=1}^{s} V\left(X_{j}\right)},
$$

for

$$
V\left(X_{j}\right)=\frac{S\left(X_{j}\right)}{\bar{X}_{j}} \quad \bar{X}_{j} \neq 0
$$

where $S\left(X_{j}\right)$ is standard deviation of a property, $X_{j}$ is the $j$-th variable.

Weights $\omega_{j}$ are called variance weights and are dependent upon the degree of variability of the property - the greater the variability of a property, the greater the weight of the assigned variable.

The last step in the construction of the global index $W_{G s}$ is based on conjugation of the transformed values; in this case, partial evaluation indices $W_{j}$ and their weights $\omega_{j}$. It is recommended to use an additive formula based on the addition of the products of normalised values of properties and their corresponding weights. The global single-value acoustic quality index of the $i$-th building is determined by the formula:

$$
W_{G s_{i}}=\sum_{j=1}^{n} W_{i j} \omega_{j} \quad(i=1,2, \ldots, r) \quad \omega_{j} \in R_{+},
$$

where $\omega_{j}$ is weight of the $j$-th uncorrelated partial index.

\section{SVD method of synthesis of building properties}

The SVD method is a technique of calculation that is in wide use in numerical linear algebra $[9,10]$. SVD is the decomposition of matrix into singular values. SVD decomposition is applicable in various fields of science such as diagnostics [11] and vibroacoustics [12, 13]. SVD is often selected as a method for solving linear problems of least squares [14]. The SVD method, similarly to factor analysis, and, in particular, the principal component analysis method (PCA) make it possible to transform the correlated data set into uncorrelated data without loss of any information.

The global acoustic quality index of a building $W_{G d}$ is related to the application of SVD to observation matrix $\boldsymbol{A}$ of all indices, either correlated or uncorrelated with each other. As a result of $\operatorname{SVD}(\boldsymbol{A})$, matrices $\boldsymbol{U}, \boldsymbol{\Sigma}$ and $\boldsymbol{V}^{T}$ are obtained with information on the independent properties of the building. Matrix we A can also be presented using the equation:

$$
\boldsymbol{A}=\sum_{i=1}^{n} \boldsymbol{u}_{i} \sigma_{i} \boldsymbol{v}_{i}
$$

where $\boldsymbol{U}_{i}$ is the $i$-th singular vector of matrix $\boldsymbol{U}$ obtained from $\operatorname{SVD}(\boldsymbol{A}), \boldsymbol{v}_{i}$ is the $i$-th singular vector of matrix $\boldsymbol{V}^{T}$ obtained from $\operatorname{SVD}(\boldsymbol{A})$, and $\sigma_{i}$ is the $i$-th singular value of matrix $\boldsymbol{\Sigma}$ obtained from $\operatorname{SVD}(\boldsymbol{A})$.

The shares of information on independent properties obtained from singular values $\sigma_{i}$ describing the variability of properties, are proposed to be used as weights of these properties. For this purpose, matrix $\boldsymbol{B}$, with uncorrelated components, is determined in the form of:

$$
\boldsymbol{B}=\sum_{i=1}^{n} \frac{\sigma_{i}}{\sum_{i=1}^{n} \sigma_{i}} \boldsymbol{u}_{i} \boldsymbol{v}_{i} .
$$

By adding the components $b_{i j}$ of matrix $\boldsymbol{B}$, the singlevalue global index for the $i$-th building is obtained:

$$
W_{G d_{i}}=\sum_{j=1}^{n} b_{i j},
$$

where $b_{i j}$ is uncorrelated partial index corresponding to the $i$-th row and $j$-th column of matrix $\boldsymbol{B}$.

\section{Verification of index synthesis methods using the example of religious buildings}

Acoustic parameters measured in six unoccupied Roman Catholic churches (reverberation time $R T$ [s], speech intelligibility index $R A S T I$, clarity $C_{80}[\mathrm{~dB}]$, sound level of external disturbances $L_{Z}[\mathrm{~dB}]$ ) were the basis for the calculation of values, developed in [5], of partial evaluation indices (the reverberation index $W_{P}$, the music sound index $W_{M}$, the speech intelligibility index $W_{Z}$, and the external disturbance index $W_{Z Z}$ ). Acoustic measurements were based on measuring impulse responses of the interiors. The MLS signal generated in the DIRAC program was used for the room excitation. An omnidirectional sound source was placed near the altar, 1.5 $\mathrm{m}$ above the floor. The microphone was placed $1.2 \mathrm{~m}$ above the floor. For calculation averaging, a dozen or so 
positions of the microphone (placed on the whole floor surface) were used. Measurements and calculations were carried out in accordance with ISO-3382 [15]. The levels of disturbing sounds $L_{Z}$ were measured by a SVAN $945 \mathrm{~A}$ sound level meter at measuring points located in the middle of each church, $1.2 \mathrm{~m}$ above the floor. During the measurements, the churches were unoccupied and their sound amplification installation was switched off.

The procedures of determination of partial indices relate to the preferred values of acoustic parameters. The reverberation index $W_{P}$ as a function of reverberation time $T_{30}[\mathrm{~s}]$, measured in church without congregation, is calculated from the equation:

$$
W_{P}=\left\{\begin{array}{c}
0 \quad \text { for } T_{30}>T_{P}+4.5 \\
-0.22\left|T_{30}-0.24 \ln \left(V_{S}\right)+0.24\right|+1 \\
\text { for } T_{30} \leq T_{P}+4.5,
\end{array}\right.
$$

where $V_{S}\left[\mathrm{~m}^{3}\right]$ is church cubic capacity, and $T_{P}[\mathrm{~s}]$ is the preferred reverberation time for Roman Catholic churches [4],

$$
T_{P}=0.24 \ln \left(V_{S}\right)-0.24 \text {. }
$$

The music sound index $W_{M}$ as a function of clarity $C_{80}[\mathrm{~dB}]$ is calculated from the equation developed on the basis of the preferred values of this parameter [4]:

$$
W_{M}=\left\{\begin{array}{l}
0 \quad \text { for }-15<C_{80}>15 \\
0.04 C_{80}+0.6 \\
\text { for }-15 \leq C_{80}<-10 \\
0.06 C_{80}+0.8 \\
\text { for }-10 \leq C_{80}<-5 \\
0.1 C_{80}+1 \text { for }-5 \leq C_{80}<0 \\
1 \quad \text { for } 0 \leq C_{80}<5 \\
-0.12 C_{80}+1.625 \\
\text { for } 5 \leq C_{80}<10 \\
-0.083 C_{80}+1.25 \\
\text { for } 10 \leq C_{80}<15
\end{array}\right.
$$

The values of the speech intelligibility index $W_{Z}$ are within the range from 0 (bad speech intelligibility) to 1 (very good speech intelligibility) and are equal to the RASTI, which has values from the same range.

The external disturbance index of the religious building $W_{Z Z}$ as a function of equivalent sound A level of external disturbance $L_{\text {Aeq }}[\mathrm{dB}]$ is given by the formula:

$$
W_{Z Z}=\left\{\begin{array}{ll}
1 & \text { for } L_{A e q} \leq 30, \\
3 /\left(L_{A e q}-27\right) & \text { for } L_{A e q}>30
\end{array} .\right.
$$

Three partial indices, $W_{P}, W_{M}$ and $W_{Z}$ are strongly correlated with each other. Index $W_{Z Z}$ is not correlated with the other indices. Linear correlation coefficients between the indices are shown in Table 1.

A single-value index, the so-called local index of selected acoustic parameters $W_{l}$, was constructed from three strongly correlated partial indices, $W_{P}, W_{M}$ and $W_{Z}$. The local index $W_{l}$ is strongly correlated with these three partial indices and not correlated with the outside disturbance index $W_{Z Z}$ (Table I).

\section{TABLE I}

Linear correlation coefficients between partial indices and the $W_{l}$ local index.

\begin{tabular}{l|r|r|r|r}
\hline \hline & \multicolumn{1}{|c|}{$W_{P}$} & \multicolumn{1}{c|}{$W_{M}$} & \multicolumn{1}{c}{$W_{Z}$} & $W_{Z Z}$ \\
\hline$W_{P}$ & 1 & & & \\
$W_{M}$ & 0.9488 & 1 & & \\
$W_{Z}$ & 0.9158 & 0.9827 & 1 & \\
$W_{Z Z}$ & -0.2280 & -0.1950 & -0.0464 & 1 \\
$W_{l}$ & 0.9893 & 0.9848 & 0.9598 & -0.2036
\end{tabular}

TABLE II

Local and global acoustic quality evaluation indices of Roman Catholic churches: St. Sebastian's Church in Strzelce Wielkie (SE), The Holiest Sacred Heart's Church in Cracow (NS), The Reformati Fathers Church in Wieliczka (RE), St. Clemens Church in Wieliczka (KL), The Jezuits Fathers Church in Cracow (JE), St. Paul Apostle Church in Bochnia (PA).

\begin{tabular}{c|c|c|c|c|c}
\hline \hline Church & Volume $\left[\mathrm{m}^{3}\right]$ & $W_{Z Z}$ & $W_{l}$ & $W_{G s}$ & $W_{G d}$ \\
\hline $\mathrm{SE}$ & 1102 & 1.00 & 1.00 & 1.0 & 0.84 \\
$\mathrm{NS}$ & 2750 & 0.39 & 0.72 & 0.60 & 0.56 \\
$\mathrm{RE}$ & 4450 & 0.37 & 0.72 & 0.59 & 0.55 \\
$\mathrm{KL}$ & 6380 & 0.58 & 0.67 & 0.64 & 0.58 \\
$\mathrm{JE}$ & 9120 & 0.58 & 0.18 & 0.32 & 0.38 \\
$\mathrm{PA}$ & 13740 & 1.00 & 0.00 & 0.36 & 0.37
\end{tabular}

Global index $W_{G s}$ values were determined from uncorrelated indices $W_{l}$ and $W_{Z Z}$ using the CMA statistical method. The weights $\omega_{1}$ and $\omega_{2}$, related to the indices $W_{l}$ and $W_{Z Z}$ are equal to 0.64 and 0.36 , respectively. The values of global index $W_{G d}$ were determined by using the method of decorrelation of partial indices $W_{P}, W_{M}, W_{Z}$ and $W_{Z Z}$. A list of values of calculated partial and global indices is shown in Table II. The values of global index $W_{G d}$ only differ slightly from the values of index $W_{G s}$ (Fig. 1). The greatest differences exist in the case of the SE building. The linear correlation coefficient between indices $W_{G d}$ and $W_{G s}$ is equal to 0.9994 .

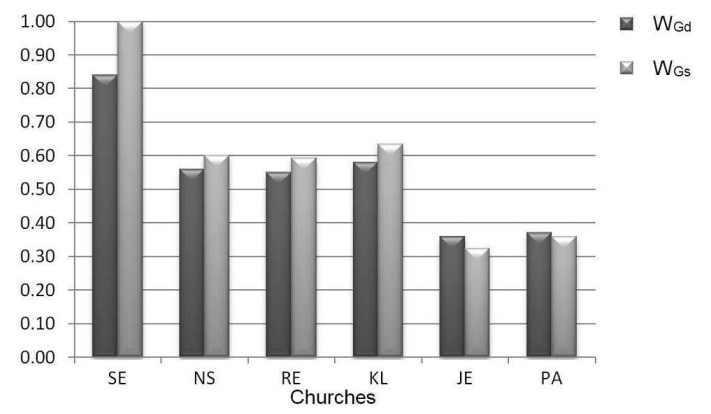

Fig. 1. Comparison of the acoustic quality of religious buildings using global indices $W_{G d}$ and $W_{G s}$.

Table III shows the correlations of global indices with partial indices. The global index $W_{G d}$ is more strongly 
TABLE III

Coefficients of linear correlation between partial and global indices.

\begin{tabular}{c|c|c|c|c|c}
\hline \hline & $W_{P}$ & $W_{M}$ & $W_{Z}$ & $r$ & $W_{Z Z}$ \\
\hline$W_{G s}$ & 0.8900 & 0.8970 & 0.9376 & 0.9082 & 0.2168 \\
$W_{G d}$ & 0.8995 & 0.9088 & 0.9475 & 0.9186 & 0.1905
\end{tabular}

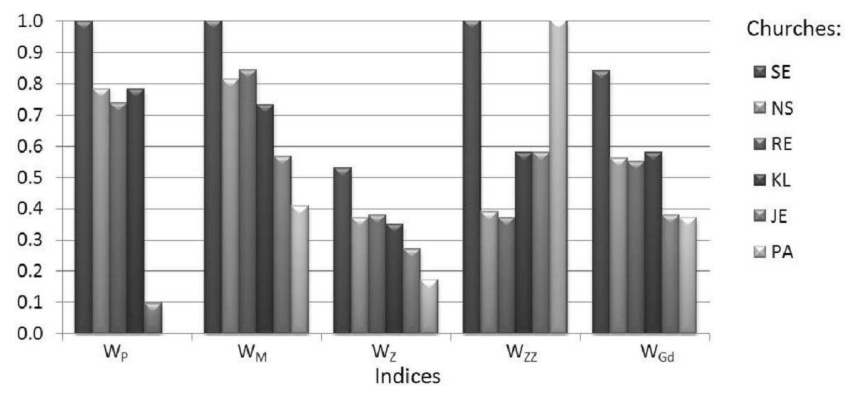

Fig. 2. Single-value evaluation of the acoustic quality of Roman Catholic churches.

correlated with partial indices $W_{P}, W_{M}$, and $W_{Z}$ than index $W_{G s}$. The average linear correlation coefficient $r$ is greater for index $W_{G d}$ as compared to $W_{G s}$.

Fig. 2 shows the single-value evaluation of acoustic quality using index $W_{G d}$ and partial indices $W_{P}, W_{M}$, $W_{Z}$, and $W_{Z Z}$. It results from Fig. 2 that the historic wooden church, SE, has the best acoustic parameters in terms of reverberation, intelligibility of speech, sound of music, and absence of external disturbances. The modern church, PA, based on an elliptical plan, has the worst acoustic parameters, excluding the level of external disturbances. The global index for this church is equal to 0.4. The second church in terms of bad acoustic parameters is the JE church. The excessive reverberation in the interior influences the bad intelligibility of speech and sound of music. Three of the studied churches among the six - NS, RE, KL - have global indices $W_{G d} \approx 0.6$ which signifies rather good conditions for fulfilment of acoustic functions. The low values of outside disturbance indices $W_{Z Z}$ are related to the location of these buildings near streets with bothersome noise from traffic.

\section{Summary and conclusions}

It was shown that the construction of a synthetic single-value index of uncorrelated partial evaluation indices may take place using the following methods: statistical - CMA and SVD. Verification of the proposed single-value evaluations was conducted on a selected group of buildings, namely Roman Catholic churches. Due to their nature, these buildings must resolve compromises between ensuring intelligibility of speech and articulation of music.

The problem of synthesis of correlated acoustic parameters and evaluation indices: of reverberation, intelligibility of speech, and articulation of music, was the subject of the author's previous works. The single-value local index of selected acoustic parameters, along with an independent index of outside disturbances served in these studies to provide a global evaluation index using the statistical method. The values of global evaluation indices obtained using the two methods discussed above are very similar and give identical results in terms of ranking of the acoustic quality of churches. However, due to the fact that the global index $W_{G d}$ obtained through SVD decorrelation, is better correlated with the initial partial indices than index $W_{G s}$ obtained using the statistical method, the evaluation conducted using index $W_{G d}$ is more reliable.

Further studies will be related to the application of indicatory evaluation methods for other public buildings and industrial buildings, as well as for a greater number of studied buildings.

\section{Acknowledgments}

The study described in this paper has been performed within the framework of statutory work of the Department of Mechanics and Vibroacoustics of AGH - 20102013, No. 3: "Prediction and experimental research of the new structures in acoustical adaptation of buildings".

\section{References}

[1] L. Beranek, Music, Acoustics and Architecture, Kruger Publ., N. York 1979.

[2] Y. Ando, Concert Hall Acoustics, Springer Verlag, Berlin, Heidelberg 1985.

[3] A. Kulowski, Room Acoustics, WPG, Gdańsk 2011.

[4] Z. Engel, J. Engel, K. Kosała, J. Sadowski, The Basis of Religious Objects Acoustics, WITE, KrakówRadom 2007, (in Polish).

[5] K. Kosała, Archiv. Acoust. 36, 545 (2011).

[6] K. Kosała, in: Proc. 15th Wibrotech 2010, Warszawa-Sękocin Stary 2010, Wyd. PW, Warszawa 2010 , p. 45.

[7] K. Kosała, Diagnostics and Structural Health Monitoring 58, 49 (2011).

[8] K. Kukuła, Zero Unitarisation Method, PWN, Warszawa 2000, (in Polish).

[9] A. Kiełbasiński, H. Schwetlick, Linear Numerical Algebra, WNT, Warszawa 1992, (in Polish).

[10] C. Lanczos, Linear Differential Operators, Van Nostad, London 1961.

[11] C. Cempel, Exploitation Problems of Machines 44, 571 (1980), (in Polish).

[12] Z. Engel, J. Engel, in: Proc. National Symposium on Machine Diagnostics, Węgierska Górka (Poland), Wyd. PŚ, Katowice 2007, p. 17.

[13] Z. Engel, J. Engel, K. Kosała, Vibroacoustic Processes: Sources, Research, Analysis, Faculty of Mechanical Engineering and Robotics AGH, Kraków 2009, (in Polish).

[14] J. Piechowicz, Acta Physica Polonica A 121, A-183 (2012).

[15] ISO 3382, Acoustics - measurement of the reverberation time of rooms with reference to other acoustical parameters, 2001. 